J. Lake Sci.(湖泊科学), 2008, 20(3): 263-270

http://www.jlakes.org. E-mail: jlakes@niglas.ac.cn

(C)2008 by Journal of Lake Sciences

\title{
长江中下游湖泊沉积物氮磷形态与释放风险关系*
}

张 路, 范成新, 王建军, 陈宇炜, 姜加虎

(中国科学院南京地理与湖泊研究所湖泊与环境国家重点实验室, 南京 210008)

摘 要: 运用聚类分析、主成分分析和相关矩阵的统计分析手段, 对长江中下游湖群共 18 个湖泊的沉积物氮磷释放风险以及 湖泊沉积物、间隙水和上覆水中氮磷形态以及其他相关地球化学参数进行分析. 草型和澡型湖泊的环境差异是造成氮磷释放 风险的主要原因. 氮磷释放风险与铁磷、藻类可利用磷、总氮、总磷、上覆水氮磷含量、间隙水氮含量、孔隙度和有机质含 量间的关系最为密切. 决定磷酸盐释放风险的主要形态磷是藻类可利用磷和铁磷，其他形态磷或者含量较低或者不易被转化 释放, 对磷酸盐释放风险影响较小. 有机磷含量对磷的释放风险没有直接决定作用, 但它与有机质含量间呈显著正相关.

关键词: 沉积物; 氮磷; 营养盐形态; 释放风险; 湖泊

\section{Nitrogen and phosphorus forms and release risks of lake sediments from the middle and lower reaches of the Yangtze River}

\author{
ZHANG Lu, FAN Chengxin, WANG Jianjun, CHEN Yuwei \& JIANG Jiahu \\ (State Key Laboratory of Lake Science and Environment, Nanjing Institute of Geography and Limnology, Chinese Academy of \\ Sciences, Nanjing 210008, P.R.China)
}

\begin{abstract}
Cluster analysis, principal component analysis and correlation matrix analysis were used to analysis the nitrogen and phosphorus release risks from sediments in 18 lakes located in the middle and lower reaches of the Yangtze River, as well as the nitrogen and phosphorus forms and related geochemical parameters from sediments, pore waters and overlying waters. The ecological difference of macrophyte and algae dominated lakes was the main reason of the difference of nitrogen and phosphorus release. The release risks were well correlated with the iron-bound phosphorus ( $\mathrm{FeP}$ ), algae available phosphorus (AAP), total nitrogen (TN), total phosphorus (TP) in sediment, the content of nitrogen and phosphorus in overlying and pore waters, porosity and organic matter content of surficial sediment. The AAP and FeP was the main phosphorus forms deciding the phosphorus release risk and other forms were in less effect on it due to the lower contents or lower transformation ability. The sediment organic phosphorus was not directly related to the phosphorus release risks but remarkably positively correlated to organic matter contents in sediment.

Keywords: Sediment; nitrogen and phosphorus; nutrients form; release risk; lake
\end{abstract}

沉积物是湖泊及其流域中营养盐及其他污染物的重要归宿和蓄积库. 沉积物中蕴藏的营养盐可以在 一定的环境条件下向上覆水体释放. 这种潜在释放能力的大小主要取决于湖泊沉积物及其上覆水体的物 理化学和生物特性的改变. 在湖泊底泥营养盐释放风险的研究中, 沉积物的物理和化学的特性(包括其含 量和地球化学形态)是影响沉积物中氮磷营养要素迁移、转化以及生态效应的重要参数.

长江中下游有许多由长江洪泛和自然演化形成的湖泊, 其中大于 $1 \mathrm{~km}^{2}$ 的 650 多个湖泊中大部分属于浅 水湖泊. 这些湖泊目前普遍受到了湖泊水质恶化, 富营养化程度加重的影响, 其生态环境和社会经济效益 严重受损. 对浅水湖泊而言，由于其更复杂的生态类型，更加频繁的水土界面营养盐交换以及更易受动力 作用的影响, 沉积物中营养盐含量和形态的差异对与水土界面交换和上覆水的营养盐含量影响机制更加复 杂 ${ }^{[1-2]}$. 虽然湖泊沉积物氮磷形态, 间隙水氮磷含量与潜在释放之间的关系已有一些研究 ${ }^{[3-7]}$, 但仍然缺乏较

* 科技部基础性工作专项(2006FY110600)和国家自然科学基金项目(40501064，40730528)联合资助. 2006-10-26 收稿; 2007-12-24 收修改稿. 张路, 男, 1975 年生, 博士, 副研究员; E-mail: luzhang@niglas.ac.cn. 

大量样本的系统综合分析. 本文从沉积物营养盐形态与释放风险之间关系的角度, 对长江中下游地区的若 干浅水湖泊进行了分类定量探讨, 尤其是对比了草、藻型两类湖泊之间释放风险的差异, 与目前仅为单一湖 泊的相关研究相比, 更能揭示湖泊氮磷形态以及相应的地球化学参数对营养盐释放风险的影响规律.

\section{1 湖泊背景及样点选择}

在长江中下游湖群选取 18 个不同环境类型、不同沉积物及水体营养盐负荷的湖泊(图 1). 其中, 洞庭 湖、鄱阳湖及洪湖的采样点在湖区中位置如图 1 所示, 由于这 3 个湖泊面积较大, 其样点仅代表采样湖 区的情况. 其余湖泊的样点一般选择在湖泊中心区. 16 个湖泊中, 共有 6 个湖泊(样点湖区)未见水草, 其 中洞庭湖和鄱阳湖未见水草可能是由于采样点区域为砂性沉积物, 且水深较深, 水草无法生长所致. 而 另外四个湖泊: 武山湖、八里湖、菜子湖和东湖也未见水草. 其余 12 个湖泊均有不同程度的水草生长. 表 1 显示了这些研究湖泊的一些环境参数和采样点位置.

表1 18 个研究湖泊位置及基本状况*

Tab. 1 Location and basic status of 18 analyzed lakes

\begin{tabular}{|c|c|c|c|c|c|c|c|c|}
\hline \multirow{2}{*}{ 湖名 } & \multirow{2}{*}{ 地点 } & \multirow{2}{*}{ 东经 } & \multirow{2}{*}{ 北纬 } & \multirow{2}{*}{ 样点水深(m) } & \multirow{2}{*}{$\begin{array}{c}\text { 透明度 } \\
(\mathrm{cm})\end{array}$} & \multirow{2}{*}{$\begin{array}{l}\text { 水草 } \\
\text { 状况 }\end{array}$} & \multicolumn{2}{|c|}{ 上覆水 $(\mathrm{mg} / \mathrm{L})$} \\
\hline & & & & & & & $\mathrm{PO}_{4}^{3-}$ & $\mathrm{NH}_{4}^{+}$ \\
\hline 西凉子湖 & 湖北咸宁 & $29^{\circ} 55^{\prime} 31^{\prime \prime}$ & $114^{\circ} 07^{\prime} 52^{\prime \prime}$ & 1.3 & 130 & 1 & 0.021 & 0.568 \\
\hline 保安湖 & 黄石大冶 & $30^{\circ} 17^{\prime} 22^{\prime \prime}$ & $114^{\circ} 43^{\prime} 20^{\prime \prime}$ & 2.1 & 200 & 1 & 0.002 & 0.129 \\
\hline 洞庭湖 & 湖南岳阳 & $29^{\circ} 21^{\prime} 33^{\prime \prime}$ & $113^{\circ} 03^{\prime} 17^{\prime \prime}$ & 0.9 & 50 & 0 & 0.034 & 0.161 \\
\hline 大官湖 & 宿松县 & $30^{\circ} 1^{\prime} 8^{\prime \prime}$ & $116^{\circ} 22^{\prime} 51^{\prime \prime}$ & 1.4 & 20 & 1 & 0.008 & 0.378 \\
\hline 鄱阳湖 & 九江星子县 & $29^{\circ} 26^{\prime} 30^{\prime \prime}$ & $116^{\circ} 2^{\prime} 21^{\prime \prime}$ & 3.2 & 50 & 0 & 0.007 & 0.157 \\
\hline 鲁湖 & 咸宁北 & $30^{\circ} 13^{\prime} 50^{\prime \prime}$ & $114^{\circ} 10^{\prime} 59^{\prime \prime}$ & 2.1 & 85 & 1 & 0.008 & 0.174 \\
\hline 梁子湖 & 湖北鄂州 & $30^{\circ} 14^{\prime} 9^{\prime \prime}$ & $114^{\circ} 36^{\prime} 57^{\prime \prime}$ & 2.0 & 160 & 1 & 0.010 & 0.449 \\
\hline 泊湖 & 宿松县 & $30^{\circ} 10^{\prime} 33^{\prime \prime}$ & $116^{\circ} 22^{\prime} 45^{\prime \prime}$ & 2.0 & 30 & 1 & 0.007 & 0.190 \\
\hline 洪湖 & 洪湖市区 & $29^{\circ} 45^{\prime} 53^{\prime \prime}$ & $113^{\circ} 22^{\prime} 37^{\prime \prime}$ & 1.5 & 150 & 1 & 0.008 & 0.235 \\
\hline 石塘湖 & 安徽安庆 & $30^{\circ} 37^{\prime} 41.8^{\prime \prime}$ & $117^{\circ} 5^{\prime} 50.4^{\prime \prime}$ & 1.1 & 60 & 1 & 0.006 & 0.295 \\
\hline 西武昌湖 & 安庆怀宁县 & $30^{\circ} 15^{\prime} 36.7^{\prime \prime}$ & $116^{\circ} 4^{\prime} 47.8^{\prime \prime}$ & 1.1 & 60 & 1 & 0.004 & 0.397 \\
\hline 策湖 & 黄冈地区 & $30^{\circ} 15^{\prime} 24^{\prime \prime}$ & $115^{\circ} 08^{\prime} 45^{\prime \prime}$ & 1.5 & 80 & 1 & 0.102 & 0.776 \\
\hline 太白湖 & 宿松县 & $29^{\circ} 58^{\prime} 11^{\prime \prime}$ & $115^{\circ} 49^{\prime} 15^{\prime \prime}$ & 2.1 & 50 & 1 & 0.011 & 1.289 \\
\hline 武山湖 & 黄冈地区 & $29^{\circ} 53^{\prime} 45^{\prime \prime}$ & $115^{\circ} 35^{\prime} 38^{\prime \prime}$ & 1.8 & 65 & 0 & 0.208 & 3.648 \\
\hline 大冶湖 & 黄石大冶 & $30^{\circ} 5^{\prime} 39^{\prime \prime}$ & $114^{\circ} 59^{\prime} 34^{\prime \prime}$ & 1.0 & 70 & 1 & 0.028 & 0.582 \\
\hline 八里湖 & 九江市郊 & $29^{\circ} 41^{\prime} 13^{\prime \prime}$ & $115^{\circ} 56^{\prime} 34^{\prime \prime}$ & 2.7 & 50 & 0 & 0.013 & 0.211 \\
\hline 菜子湖 & 安徽桐城 & $30^{\circ} 50^{\prime} 9^{\prime \prime}$ & $117^{\circ} 2^{\prime} 7^{\prime \prime}$ & 1.0 & 15 & 0 & 0.017 & 0.438 \\
\hline 东湖 & 武汉市区 & $30^{\circ} 33^{\prime} 16^{\prime \prime}$ & $114^{\circ} 23^{\prime} 38^{\prime \prime}$ & 3.8 & 80 & 0 & 0.037 & 0.734 \\
\hline
\end{tabular}

* 1 表示样点附近肉眼可见水草; 0 表示肉眼不可见水草.

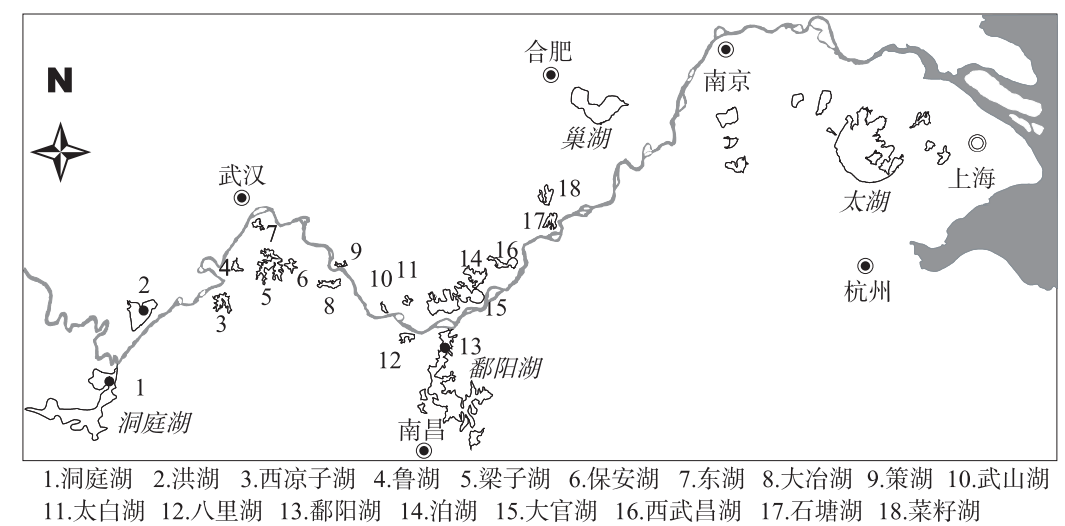

图 1 长江中下游湖群及样点示意图

Fig.1 Sketch map of sampling locations along the middle and lower reaches of the Yangtze River 


\section{2 研究方法}

\section{1 沉积物释放实验}

对柱状沉积物进行原柱样控温培养, 获得沉积物的氮磷营养盐释放速率 ${ }^{[8]}$.

释放速率 flux 按下式计算 ${ }^{[8]}$ :

$$
f l u x=\left[V\left(C_{n}-C_{0}\right)+\sum_{j=1}^{\mathrm{n}} V_{j-1}\left(C_{j-1}-C_{\mathrm{a}}\right)\right] / S \cdot t
$$

其中, flux 为释放速度 $\left(\mathrm{mg} /\left(\mathrm{m}^{2} \cdot \mathrm{d}\right)\right) ; V$ 为柱中上覆水体积 $(\mathrm{L}) ; C_{\mathrm{n}} 、 C_{0} 、 C_{j-1}$ 为第 $n$ 次、0 次(即初始)和 $j-1$ 次 采样时某物质含量 $(\mathrm{mg} / \mathrm{L}) ; C_{\mathrm{a}}$ 为添加水样中的物质含量 $(\mathrm{mg} / \mathrm{L}) ; V_{j-1}$ 为第 $j-1$ 次采样体积 $(\mathrm{L}) ; S$ 为柱样中水 一沉积物接触面积 $\left(\mathrm{m}^{2}\right) ; t$ 为释放时间 (d). 所计算的 $\mathrm{NH}_{4}{ }^{+}$和 $\mathrm{PO}_{4}{ }^{3-}$ 潜在释放均为 $3 \mathrm{~d}$ 平均表观释放速率, 分 别以小写参数符号的形式表示为 nflux 和 pflux (下同).

\section{2 表层沉积物生物地球化学参数}

沉积物释放实验结束后, 将沉积物柱样按 $1 \mathrm{~cm}$ 厚度分层. 取 $10 \mathrm{~g}$ 左右的表层沉积物进行以下分析：

形态磷 ${ }^{[9]}$ : 利用连续提取法, 对沉积物中的形态磷进行分级提取. 由于闭蓄态磷在生物地球化学循 环中参与磷的短时相的交换转化的可能性非常小，虽进行了提取，但数据相关分析时未予考虑. 因此主 要考虑了如下五种形态磷：铁结合态磷 $(f e p, \mathrm{mg} / \mathrm{kg})$ 、可交换态磷 $(l p, \mathrm{mg} / \mathrm{kg})$ 、铝结合态磷 $(a l p, \mathrm{mg} / \mathrm{kg})$ 、钲 结合态磷 (cap, $\mathrm{mg} / \mathrm{kg}$ )、有机磷 (orgp, $\mathrm{mg} / \mathrm{kg}$ ).

对沉积物样品同时测定：藻类可利用磷 ${ }^{[10]}$, 即利用碱性提取法, 对沉积物中可潜在供藻类利用的磷 量 $($ aap, $\mathrm{mg} / \mathrm{kg})$ 进行分析. 利用强酸消解法测定沉积物中的总磷 $(t p, \mathrm{mg} / \mathrm{kg})$ 、总氮 $(t n, \mathrm{mg} / \mathrm{kg})$; 用高温灼烧 法测定有机质含量(以烧失量计, 表示为 $l o i, \%) ; 105^{\circ} \mathrm{C}$ 下烘干法测定沉积物孔隙度(por, \%).

\section{3 上层间隙水生物地球化学参数}

将分层的表层沉积物样进行离心和过滤, 获取间隙水, 立即测定间隙水中酸碱度 $(p h)$; 利用比色分析 法测定溶解性磷酸盐 $(p o 4 p)$ 、溶解性氨态氮 $(n h 4 p)$; 利用总有机碳分析仪 $(1020 \mathrm{~A})$ 同步测定了间隙水中溶 解性总有机碳 $(t o c)$ 和溶解性总无机碳( $t i c)$.

\section{4 湖泊水体基本参数}

湖水透明度(以透明度盘深度 Secchi Depth 表示, $s d, \mathrm{~cm}$ )；用比色分析法测定溶解性磷酸盐( po4w， $\mathrm{mg} / \mathrm{L})$ 、溶解性氨态氮 $(n h 4 w, \mathrm{mg} / \mathrm{L})$; 电导率仪测定水体中的电导率 $(e c, \mu \mathrm{s} / \mathrm{cm})$. 另外, 考察了采样点有无 水草(plant, 有水草赋值为 1 , 无水草赋值为 0$)$.

\section{3 结果}

\section{1 营养盐及释放风险的地球化学参数聚类分析}

将 18 个长江中下游湖泊的生物地球化学特征参数分为 4 类: 1)表层沉积物: lp, alp, cap, orgp, fep, aap, $t p, t n, l o i, p o r ; 2)$ 上层间隙水: $p h, p o 4 p, n h 4 p, t o c, t i c ; 3)$ 水土界面营养盐潜在释放: pflux, nflux; 4)湖泊水体 基本参数: $s d, p o 4 w, n h 4 w, e c$, plant. 从生物地球化学角度出发, 这 22 个参数都是与营养盐在水土界面上 的潜在释放风险和沉积物的营养盐含量、形态和转化相关联的参数. 将参数值构建成一个 $22 \times 18$ (参数 $\times$ 样点数)的矩阵, 并对其进行聚类分析(组间均值联接法, SPSS 10.0). 在聚类分析中, 未将采样点有无水草 (plant)作为系统变量, 以排除该变量对聚类分析可能产生的影响.

聚类分析结果(图 2)显示: 这 18 个湖可以分成具有显著差异的两组(组 1 和组 2), 而组 2 又可以分 为较小差异的两组(组 3 和组 4). 在对湖泊分组的生物地球化学意义进行讨论时我们发现, 组 1 的 4 个 湖泊: 武山湖、八里湖、菜籽湖和东湖所在样点都未见水草生长. 在全部 18 个研究湖泊中, 未观察到 有水草存在的湖泊共有 6 个(表 1), 除了组 1 表示的 4 个无水生植被的湖泊外，洞庭湖和鄱阳湖在采样 点附近区域也未观察到水生植被. 但是，考虑到洞庭湖和鄱阳湖的采样点没有水草可能与砂性底质和 水深有关, 因此, 可以认为组 1 代表的是没有水草生长的藻型湖泊, 其显著特点是沉积物总磷和藻类 可利用磷含量均较高, 属于高营养本底的湖泊. 而组 2 分为组 3 和组 4, 其中组 3 有 6 个湖泊: 泊湖、 大冶湖、太白湖、西凉子湖、鲁湖和洪湖, 组 4 有 8 个湖泊: 石塘湖、策湖、大官湖、鄱阳湖、梁子 
湖、西武昌湖、保安湖、洞庭湖. 该组除洞庭湖和鄱阳湖未见水草外, 其余湖泊为草型湖泊, 且沉积物 的营养本底较底.

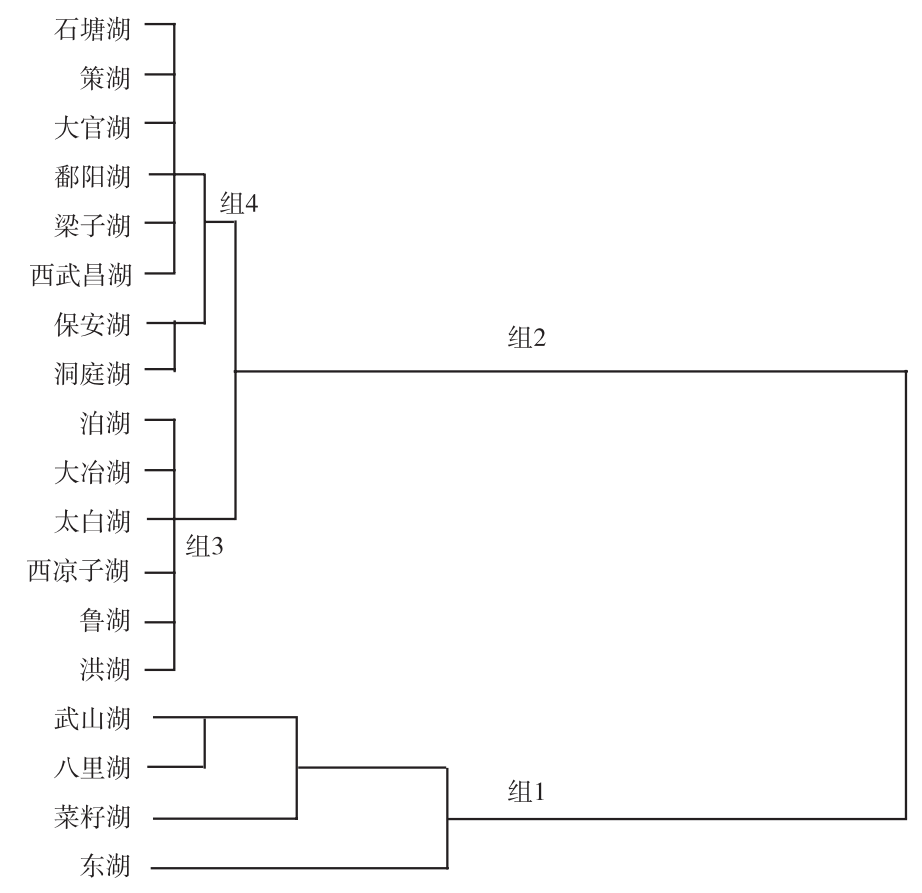

图 2 组间均值联接聚类分析法系统树

Fig.2 Systematic tree of between group linkage cluster analysis

\section{2 潜在释放与沉积物、间隙水和上覆水氮磷含量}

氮磷潜在释放均以沉积物高氮磷营养本底的武山湖为最高, 同样该湖泊沉积物间隙水中的可溶性 氮磷含量也相对较高. 同时由于沉积物的潜在释放导致该湖泊的上覆水溶解性氮磷含量在 18 个湖泊中 最高(图 3). 从图 3 中看出, 组 1 四个湖泊的沉积物总磷和总氮含量平均值为 $1187 \mathrm{mg} / \mathrm{kg}$ 和 $2286 \mathrm{mg} / \mathrm{kg}$. 与此相比较, 组 3 组 4 共 14 个湖泊沉积物的总磷和总氮含量平均值仅分别为 $625 \mathrm{mg} / \mathrm{kg}$ 和 $1122 \mathrm{mg} / \mathrm{kg}$. 由于沉积物中的氮磷首先要转化成间隙水中的可溶性氮磷才能在浓度梯度或者动力作用下向上覆水方向移 动, 形成潜在释放, 介于沉积物和上覆水之间的媒介一间隙水的含量就非常重要. 从结果看, 组 1 湖泊的间 隙水中溶解性氮磷平均含量分别为 $4.23 \mathrm{mg} / \mathrm{kg}$ 和 $0.02 \mathrm{mg} / \mathrm{kg}$, 而组 3 和组 4 的湖泊为 $1.84 \mathrm{mg} / \mathrm{kg}$ 和 $0.02 \mathrm{mg} / \mathrm{kg}$. 由于组 1 湖泊的沉积物和间隙水氮磷含量均高于组 3 和组 4 , 因此可以预计组 1 湖泊的氮磷潜在释放 可能高于组 3 和组 4 . 实验结果证实了这样的猜测: 组 1 四个湖泊的磷酸盐和氨态氮的潜在释放速率 平均为 $0.41 \mathrm{mg} /\left(\mathrm{m}^{2} \cdot \mathrm{d}\right)$ 和 $13.2 \mathrm{mg} /\left(\mathrm{m}^{2} \cdot \mathrm{d}\right)$, 显著高于组 3 和组 4 的 $0.18 \mathrm{mg} /\left(\mathrm{m}^{2} \cdot \mathrm{d}\right)$ 和 $3.33 \mathrm{mg} /\left(\mathrm{m}^{2} \cdot \mathrm{d}\right)$. 因此, 从这个结果可以直观地认为沉积物氮磷含量与氮磷潜在释放间有一定的对应关系.

\section{3 湖泊磷释放与磷含量结果}

按聚类分析结果, 将 18 个湖泊分为三组. 图 4 显示了各湖泊组磷释放风险与沉积物中 $f e p 、 a a p 、 t p$ 以及间隙水磷 $p o 4 p$ 、上覆水磷 $p o 4 w$ 的比较结果. 四个藻型湖泊的磷释放通量高于组 3 和组 4 , 组 3 和组 4 的释放风险中值相近, 但组 3 各湖泊的释放风险差异大于组 4. fep 含量结果为组 $1>$ 组 $3>$ 组 4 , 但组 1 四 个湖泊含量差异较大. a a p 和 $t p$ 含量规律类似与 fep. 但组 1 的数据分离度小于 fep. 上覆水磷酸盐含量各 组间差异并不显著, 但间隙水中磷酸盐含量表现为组 $1>$ 组 $3 \approx$ 组 4 . 

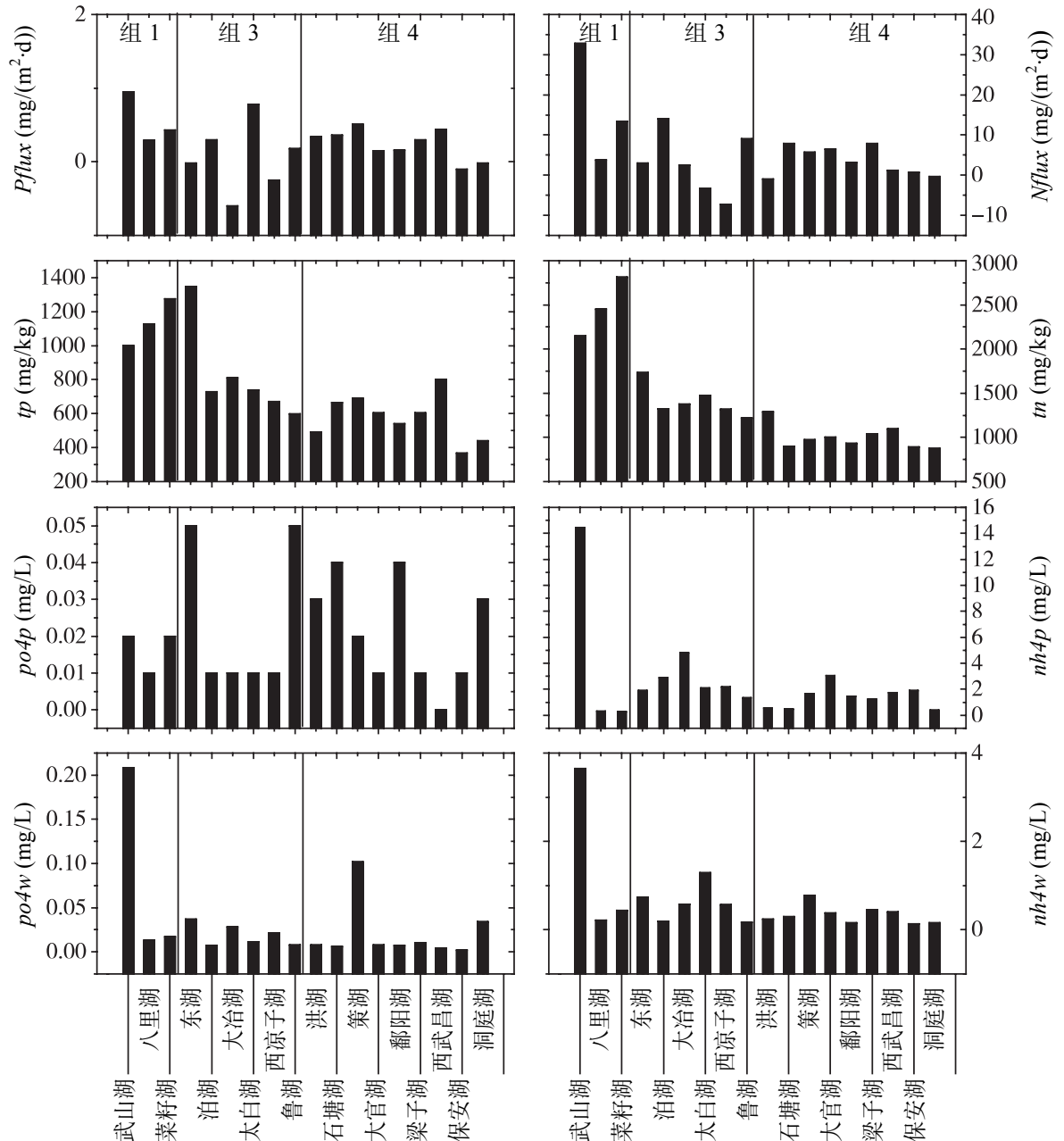

图 3 氮磷潜在释放与沉积物、间隙水、上覆水氮磷含量

Fig.3 Potential release of nitrogen and phosphorus and their contents in sediments, pore waters and ovelying coaters
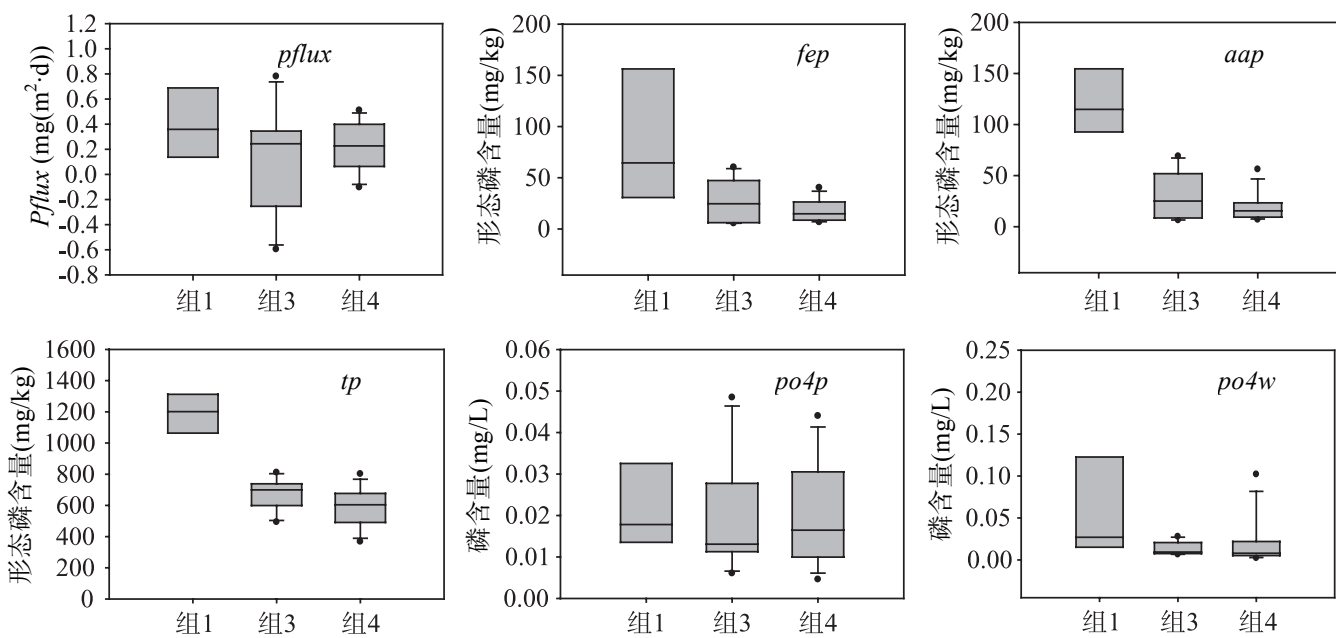

图 4 三组不同湖泊磷释放潜力和沉积物、间隙水和上覆水磷含量比较箱式图

Fig.4 Comparison of potential release of phosphorus and contents of phosphorus in sediments, pore waters and overlying waters 


\section{4 讨论}

\section{1 沉积物氮磷潜在释放参数的因子分析}

在进行相关分析前, 有必要对 22 项与潜在释放有关的生物地球化学参数进行因子分析，即运用主 成分分析法, 篮选出与沉积物潜在释放相关联的参数. 从理论上说, 沉积物的营养盐含量、形态及相应的 物理化学条件是影响营养盐潜在释放的条件, 按照连续提取法对沉积物形态磷进行分级提取后, 沉积物 形态磷可以分为潜在释放的磷源: 可交换态磷、铝磷、铁磷和有机磷难以利用的磷源: 钻磷等. 而沉积物 的有机质含量、间隙水氮磷含量以及酸碱度和有无沉水植物等条件. 可能是影响沉积物氮磷潜在释放的 因素.

图 5 表示这些生物地球化学特征参数在经主成分分析(经最大方差法旋转和 Kaiser 均一化, Varimax with Kaiser normalization)提取主要因子后, 分为 2 个主成分(主成分 1 和主成分 2). 其中, 反映释放风 险的 $n f l u x$ 和 $p f l u x$ 的两个参数与 $f e p 、 a a p 、 t n 、 t p 、 p o 4 w 、 n h 4 w 、 n h 4 p 、 p o r$ 和 $l o i$ 在同一个主成分 1 中. 这 11 个参数构成了影响氮磷营养盐释放风险的主要生物地球化学参数. 从主成分 1 中可以看出, $f e p$ 和 $a a p$ 提取的因子信息最大，从统计角度出发，可以认为这两个参数对该主成分的贡献最大，而 $t n$, $t p, p f l u x$ 和 $n f l u x$ 提取的因子信息也较大，说明这四个参数也是该主成分的重要组成信息. 而 $l o i 、 p o r 、$ $p o 4 w 、 n h 4 w 、 n h 4 p$ 对该主成分的构成也有一定的贡献，但不起决定作用. 主成分 2 包含的参数信息，如 $a l p 、 l p 、 c a p$ 和 $\operatorname{org} p$ 等与组 1 中磷释放风险的参数分属两个主成分, 可能说明了这些形态磷对磷的释 放风险不起决定作用. 从主成分 2 中还能看出, 表征湖泊有无水草的特征参数 plant 出现在该组中, 是 否也说明了有无水草的生长与湖泊沉积物的营养盐释放风险间并不一定有必然的联系. 而主成分 2 中 的 $p h$ 可能也说明水体酸碱度与沉积物的营养盐释放风险间的联系并不十分密切，同样的结论在太湖的 梅梁湾和东太湖这两个湖区的沉积物营养盐释放比较研究中得到 ${ }^{[4]}$. 虽然也有一些研究报道认为间隙 水酸碱度与沉积物营养盐释放间存在联系 ${ }^{[11]}$, 但同样也有许多研究对此结论持怀疑态度 ${ }^{[12]}$. 主成分 2 中 plant 和 $s d, p h$ 具有较高的因子信息可能预示着水草可能对控制湖水透明度和酸碱度有一定的关联 作用.

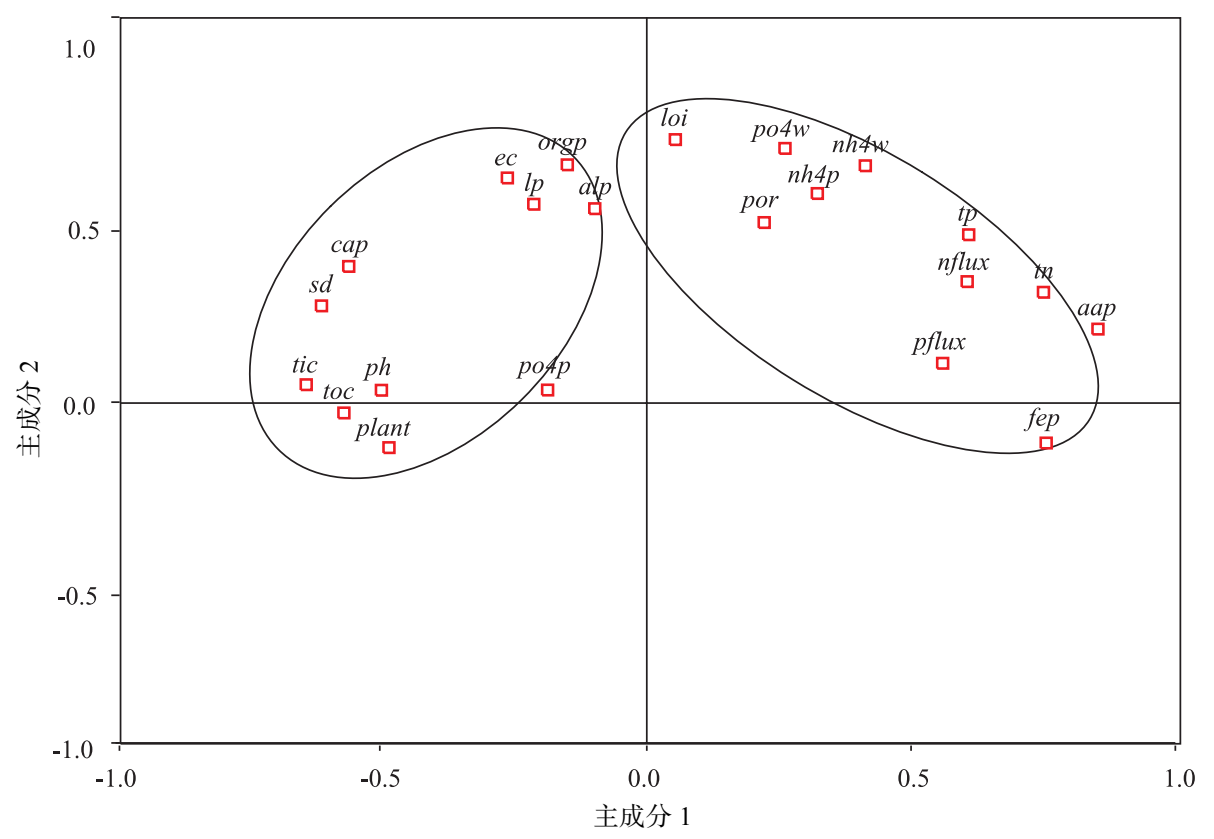

图 5 营养盐及释放风险生物地球化学参数主成分分析

Fig.5 Principal component analysis on biogeochemical parameters of nutrients and their release risks 
在进一步分析相关关系前, 对主成分分析的可靠性进一步分析, 分析结果显示主成分 1 中的参数的公 因子方差提取比例都超过了 $50 \%$ (除 pflux 和 por)，且 a a p 在主成分 1 中提取的公因子方差最高; 而主成分 2 的公因子方差提取比例均未超过 $50 \%$. 这预示着相比主成分 2 ，主成分 1 的因子提取具有更高的可靠性.

\section{2 沉积物磷形态与磷释放的相关分析}

已有研究表明, 影响磷潜在释放的形态磷主要是可交换态磷、铅磷、铁磷和一部分有机磷. 前期研 究 ${ }^{[9]}$ 认为虽然可交换态磷和铝磷是容易释放或者容易转化成易释放磷的形态磷, 但是由于沉积物中这两 个形态磷的含量很低, 不能决定磷的潜在释放. 而具有易转化性和较高含量的铁磷和一部分有机磷是决 定沉积物间隙水磷酸盐含量以及磷释放的形态. 在这 18 个湖泊的研究中, 发现了类似的结论. 虽然这 18 个湖泊具有不同的营养盐负荷、不同的环境类型, 但是可交换态磷和铝磷的含量均很低, 约比铁磷低 2-3 个数量级. 因此, 这些易转化的磷形态与磷释放之间的相关关系比较弱.

考虑到聚类分析得到的结果一一组 1 和组 $3 、 4$ 间具有较大的差异, 因此在分析这些形态磷和潜在释 放之间关系时, 主要考虑了组 3 和组 4 的 14 个湖泊, 相关分析结果仅考虑了这些有水草生长的湖泊.

与磷的潜在释放关系最为密切的是藻类可利用磷 (aap), 皮尔逊相关系数 $r=0.78$, 显著因子 $p=0.002$, 属于极显著正相关. 由于铁结合态磷( fep ) , 也基本能反映 $a a p$ 的含量, 因此, 磷的潜在释放与 $f e p$ 间也呈 极显著正相关. 其他的形态磷均未发现显著的相关关系. 这个结论与太湖几个湖区得到的结论基本类似. 因此, 对于这些湖泊而言, 决定磷潜在释放的沉积物形态磷组分主要是铁磷, 用藻类可利用磷含量来指 示磷潜在释放的量也具有较高可靠性.

\section{3 沉积物氮磷形态与潜在释放特征参数的相关关系}

将聚类分析中构建的 $22 \times 18$ 的矩阵进行相关分析, 其中未将水草参数考虑在相关关系分析中. 从分 析结果看(表 2), 与磷的潜在释放显著正相关的主要参数有 $n f l u x 、 f e p 、 a a p 、 t p 、 p o 4 w$ 和 $n h 4 w$ ，显著负 相关的有 $t o c 、 t i c$. 与氮的潜在释放显著正相关的有 $a a p 、 t p 、 t n$ ，极显著正相关的有 $n h 4 p 、 p o 4 w 、 n h 4 w$. 由于 $p o 4 w$ 和 $n h 4 w$ 之间的极显著正相关关系, 所以氮的潜在释放与 $p o 4 w$ 有关, 但显然水体中的磷酸盐 含量 $(p o 4 w)$ 高低不是决定氮的潜在释放的因素; 同样的，与磷的潜在释放显著正相关的 $n h 4 w$ 也不能说明 水体中溶解性氨氮 $(n h 4 w)$ 是决定沉积物磷释放的因素. 与此类似，由于总磷 $(t p)$ 、藻类可利用磷 $(a a p)$ 和总 氮 $(t n)$ 之间存在极显著的相关性，因此，造成了沉积物氮磷潜在释放与这些参数间的表观相关性.

从与沉积物氮磷的潜在释放相关的参数看, 主要是沉积物氮磷营养盐的含量决定的. 而铁结合态磷 与磷潜在释放间相对较好的相关系数表明用铁结合态磷表征磷的潜在释放是比较合乎规律的, 其次是用 aap 来表征的藻类可利用磷. $p o 4 w$ 与磷潜在释放间的正相关关系可能是释放的结果. 可以推测, 如果水体 中的磷酸盐含量与沉积物的潜在释放相关, 那么这样的湖泊水体中的磷酸盐的来源可能与内源性磷关系 更大，而不是外源性磷.

从表 2 还可以预示出: 钙结合态磷 $(c a p)$ 与其他参数均没有显著相关, 仅与电导率 $(e c)$ 有一定的关联. 由于钲结合态磷是非常不活跃的形态磷，主要受热力学中的溶解沉淀平衡控制，而磷酸钻的溶度积 $K_{s p}$ 为 $1.6 \times 10^{44}$, 很难被溶解, 因此对间隙水、上覆水磷以及磷潜在释放的影响较小, 一般来说钙结合态磷主要 表现出沉积埋藏的特性. 有机磷 $(o r g p)$ 与表征有机质含量的 $l o i$ 极显著正相关, 说明这两个参数表征的是 有机污染而不是营养盐的污染. 类似的结论在太湖也被发现.

间隙水的氨氮含量与氮的潜在释放极显著相关, 而间隙水磷的含量与潜在释放间没有显著相关关系, 是否预示着间隙水中氮的迁移转化更多的受浓度梯度的控制, 而磷在间隙水中的迁移并非只受浓度梯度 的控制, 在这个过程中有机磷矿化等地球化学过程可能也起到了很重要的作用 ${ }^{[2]}$.

\section{5 结论}

将氮磷释放风险及与之相关的沉积物、间隙水和上覆水中氮磷形态、含量及其它地球化学参数进行 聚类分析, 认为湖泊环境类型的差异是造成这些因素差异的最主要原因. 氮磷释放风险与铁磷、藻类可 利用磷、总氮、总磷、上覆水氮磷含量、间隙水氮含量、孔隙度和有机质含量间的关系最为密切. 决定 磷酸盐释放风险的主要形态磷是藻类可利用磷，其次为铁磷. 沉积物中有机磷与有机质呈显著正相关, 
而其他形态磷或者含量较低或者不易被转化释放, 对磷酸盐释放风险影响较小.

表 2 沉积物氮磷形态与潜在释放特征参数相关关系*

Tab. 2 Correlation between nitrogen and phosphorus forms and potential release parameters

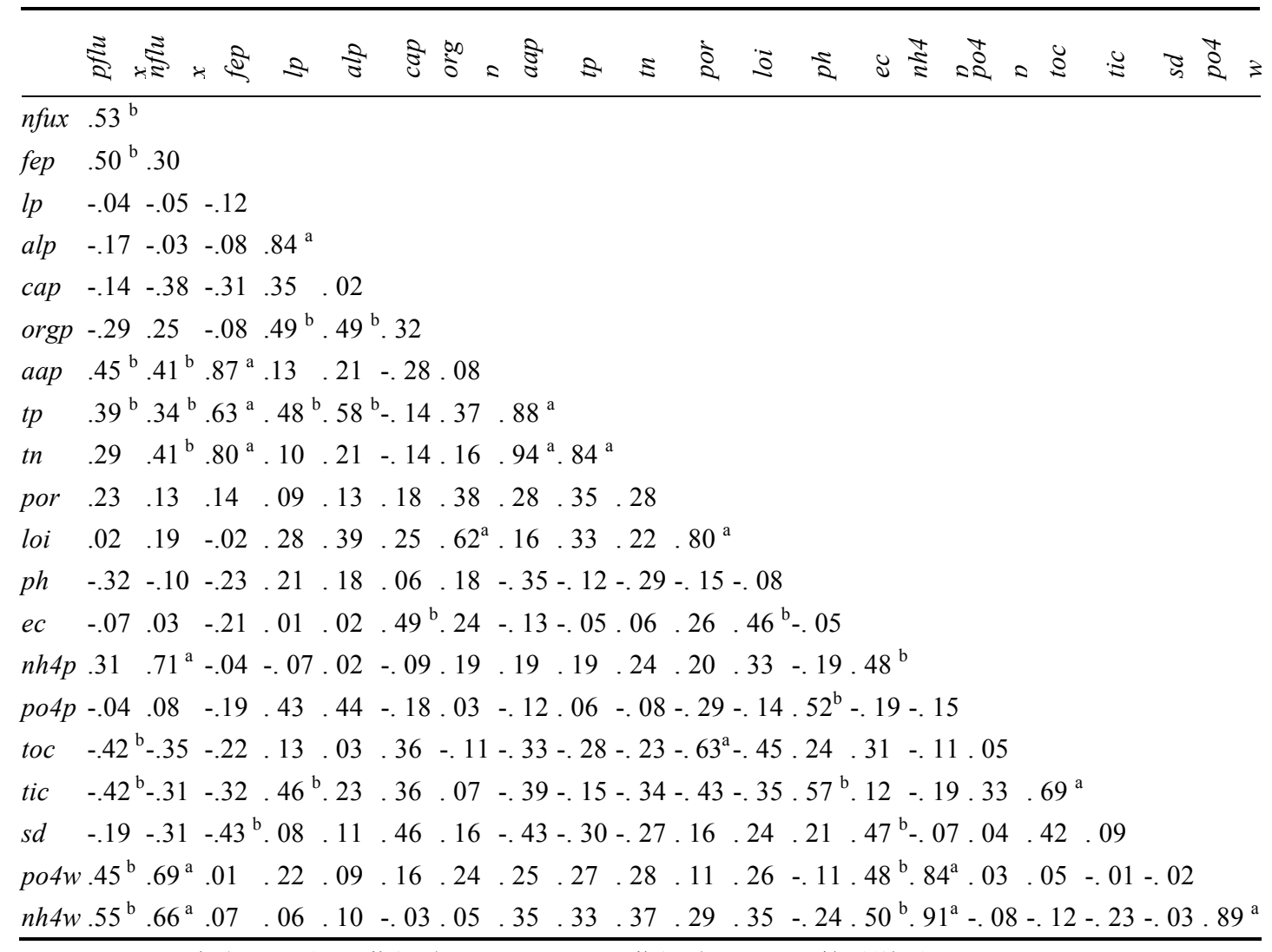

*Pearson 双尾检验； $a$ : 极显著相关 $p<0.01 ； \quad$ : 显著相关 $p<0.05$; 符号说明见 1.2 .

\section{6 参考文献}

[1] Wetzel RG. Limnology. Lake and River Ecosystems (Third Edition). San Diego: Academic Press, 2001: 625-627.

[2] 范成新, 张 路, 秦伯强等. 风浪作用下太湖悬浮态颗粒物中磷的动态释放估算. 中国科学( D辑), 2003, 33(8): 760-768.

[3] 徐 清, 刘晓端, 王辉锋等. 密云水库沉积物内源磷负荷的研究. 中国科学(D辑), 2005, 35(增 I ): 281-287.

[4] 张 路, 范成新, 王建军等. 太湖水土界面氮磷交换通量的时空差异. 环境科学, 2006, 27(8): 1537-1543.

[5] 范成新, 杨龙元, 张 路. 太湖底泥及其间隙水中氮磷垂直分布及其相互关系分析. 湖泊科学, 2000, 12(4): 359-366.

[6] 范成新, 张 路, 秦伯强等. 太湖沉积物一水界面生源要素迁移机制及定量化一- I.氨态氮释放速率的空间差异及源汇 通量. 湖泊科学, 2004, 16(1): 10-20.

[7] 黄清辉, 王子健, 王东红等. 太湖表层沉积物的磷吸附容量及其释放风险评估. 湖泊科学, 2004, 16(2): 97-104.

[8] 范成新, 张 路, 杨龙元等. 湖泊沉积物氮磷内源负荷模拟. 海洋与湖沼, 2002, 33(4): 370-377.

[9] 张 路, 范成新, 池俏俏等. 太湖及其主要人湖河流沉积磷形态分布研究. 地球化学, 2004, 33(4): 423-432.

[10] 张 路, 范成新, 朱广伟等. 长江中下游湖泊沉积物生物可利用磷分布特征. 湖泊科学, 2006, 18(1): 36-42.

[11] Xie LQ, Xie P, Tang HJ. Enhancement of dissolved phosphorus release from sediment to lake water by Microcystis blooms-an enclosure experiment in a hyper-eutrophic, subtropical Chinese lake. Environmental Pollution, 2003, 122: 391-399.

[12] Gibson CE, Wang G, Foy RH et al. The importance of catchment and lake processes in the phosphorus budget of a large lake. Chemosphere, 2001, 42: 215-220. 